\title{
Type Preference of Female Character and Color Choice in Manicure Based on the Eye Movement Technique
}

\author{
Bo Zhao ${ }^{1, *}$, Yafeng Nan ${ }^{1}$, Qiao Li Wang ${ }^{1}$ \\ ${ }^{1}$ LiaoNing Technical University \\ *Corresponding author. Email: 6589054@qq.com
}

\begin{abstract}
In the category of consumer psychology, color psychology and personality psychology complement each other, while how a specific personality characteristic affects the color choice of female consumers is still unknown. In order to further explore this problem, this paper proposes a method to predict user decision prediction based on eye movement technique. The author explored the nail art pictures preference relations of four personality types and 4 kinds of colors. The research findings from this study have important implications for user-centered marketing recommendation and advertising image design. Based on the consumers' preference, service personnel can infer consumers' personality type according to their behavior, recommend products that conform to consumers' color preference.
\end{abstract}

Keywords : Personality type, Color preference, Eye movement technique, Consumer psychology

\section{INTRODUCTION}

In recent years, the research on the role of experiential emotion in consumption decision has been paid more and more attention in the field of marketing [1]. Enterprises make color preference prediction according to consumers' personality in the market targeting and improve consumers' experiential emotional cognition of products, enterprises can greatly improve customers' favorable impression of products or brands, save service time and reduce the cost of serving customers [2]. As a key experiential factor in consumers' purchasing process, color preference plays an important role in consumers' decision-making gradually. Color vision leads to physiological response of people through color stimulation. According to the physical feelings that color brings to people, color can be divided into cool color series, warm color series, and neutral color series. Warm colors include red, orange and yellow. Cool colors include blue, purple and green. Neutral colors include black, gray and white. Color preference is due to the emotional response to objects related to color. Consumers choose colors according to the physiological response formed by color stimulation. And consumers tend to choose the color they like and avoid the color they hate [3]. When discussing the general characteristics and related factors of color preference in healthy people,
Marzolf and Kirchner [4] find that personality traits are the main factors influencing color preference. The abstract color preference in healthy adults is blue and red, which is affected by biological, psychological and social factors.

The researchers conclude that gender plays a prominent role in color preference. Granger's [5] experimental results show that gender difference exist in masculine feminine, whereas no significant cultural difference is found between British and Chinese observers. Fang, Su and Li [6] study the color preference and psychological cognitive habits of college students on underwear, and there are significant gender differences between college students on underwear color and psychological cognitive habits. The studies show that women have higher awareness of color and their preference in color selection are more varied, in other words, females have more focus on color than males, and have greater value to be explore.

Therefore, this paper aims at the female population, takes nail as the research scene, and selects the stable internal factor as the research variable to carry out the research on the color preference of individuals with different personality types. Moreover, the application of eye movement technology improves the objectivity and accuracy of experimental data in the study. 


\section{THEORETICAL BACKGROUND AND HYPOTHESES}

\subsection{Personality}

Personality is not only genetic, but also is affected by acquired life experience. It is a comprehensive embodiment of biological and social nature. At the same time, personality is the individual's stable attitude towards reality and the individual psychological characteristics of habitual behavior [7]. As the core of individual psychological characteristics, the personality characteristics of consumers reflect personality and reveal the inherent nature of individual differences of consumers, which plays the most crucial guiding role in behavior [8]. According to the distinction in individual behavior habits, style and thinking mode, personality is divided into four types: active type, strength type, perfect type and peaceful type [9].

\subsection{Personality and Color}

Based on the relationship between personality and color, there are similar behavioral characteristics corresponding relationships between different colors and personality. Liu [10] studies the relationship between personality and color from the perspective of metaphor and found that there is a metaphorical relationship between color and the personality that represented. Colors that represent certain behavioral traits correspond to personality that have the same behavioral traits. In addition to the three typical colors of warm color, cool color and neutral color, nude color which is similar to the nail color or transparent nail effect in manicure activities is the remaining choice of all possible options. The nude color series is added into the study to ensure the rigor of the experiment. Therefore, warm color fastens the behavior characteristic that has consistent with active disposition: extroversion, active; the cool color series and the strength type character display for the extroversion, the connotation, the grace; the neutral color is introverted, pure and peaceful. Nude and perfectionist are introverted, low-key and silent. However, color can arouse consumers' association of color personality, and preference for certain color will generate preference for brands and products with such color [11] It can be inferred that, consumers prefer color that represent the same behavioral characteristics as consumers' personality at the time of color decision. Based on the consistency correspondence, the hypotheses are put forward.

Hypothesis 1: Active type personalities prefer warm colors (red, orange, yellow). For the active type consumers, warm color fixation time and fixation count are significantly higher than those of other colors.

Hypothesis 2: Strength type personalities prefer cool color series (blue, green, purple). For the strength type consumers, cool color fixation time and fixation count are significantly higher than those of other colors.

Hypothesis 3: Perfect type personalities prefer nude color (nude skin, nude pink, transparent). For the perfect type consumers, nude color fixation time and fixation count are significantly higher than those of other colors.

Hypothesis 4: Peaceful type personalities prefer neutral color (black, white, gray). For the peaceful type consumers, neutral color fixation time and fixation count are significantly higher than those of other colors.

Hamel, Guyader, Pellerin and Houzet [12] find that a slight influence of color on eye movements is measured, and only the number of attractive regions for color stimuli is slightly higher than for grayscale stimuli. However, a luminance-based saliency model predicts the eye positions for color stimuli as efficiently as for grayscale stimuli.

Color affects the selective aspects of attention [13]. Consumers have disparate levels of visual sensitivity to different colors. Therefore, we propose that consumers have a consistent perceptual order for the presentation of color series in vision.

Hypothesis 5: Four color series have a sequential attraction to consumers. That is, consumers' the entry time to color series is in order.

\section{RESEARCH METHOD}

This study takes nail color selection as the experimental design scenario. A total of 24 stimulus images were included in the formal experimental materials which were played in 4 groups. Each group consists of 6 images, and a blank page was inserted between the groups to remind them to rest.

30 college females were randomly selected to rate the psychological acceptance degree of different colors in the experimental materials. Each item is rated on a 5- point Likert scale, indicating that the scale has good internal consistency and high reliability.

The iView X HED head-mounted eye-movement tracking device of SMI was used in the experiment.

The personality questionnaire, according to the definition of Florence Littauer in 1996, divides personalities into four type: strength, active, perfect and peace. The mature personality test questionnaire consists of 40 questions to determine the personality type of the participants.

The participants in this study selected randomly 42 students who have never been affected by nail color selection in a Chinese public university (right-handed), ages ranging from 18 to 29 years $(\mathrm{M}=21.3, \mathrm{SD}=1.7)$ and the experiment were limited to female whose vision was over 1.0 without color blindness or color weakness. 
The participants were informed to read a scenario after completing the personality test questionnaire. "Now we invite you to do a pure color nail for free. Please sit $60 \mathrm{~cm}$ away from the computer screen and conduct eye movement five-point calibration test." Participants viewed 36 pure color nail images that are played on the computer automatically and finally selected their favorite color images from paper showing the same content.

Statistically, the characteristic types of all the participants included 13 active type, 8 strength type, 9 perfect type and 12 peaceful type. The participants were all female college students. ANOVA was used to test demographic variables had no effects on fixation time and fixation count.

\section{RESULTS}

\subsection{Analysis of Eye Movement Index between Personality Type and Color Type}

In the same type of advertisement, there is a significant difference in the fixation time between the advertisements in which consumers finally choose to buy. That is, the longer the staring time is, the more likely the consumers are to buy [14].

The fixation of the one character on four color series

This paper aims to find the fixation preference of consumers with the same personality type on the pictures of nails with four different colors. Based on the classification of four personality types, univariate analysis of variance is used to analyze the fixation of the same personality type on nails with four colors.

Take consumers with active personality type as the research object, the results of one-way ANOVA of fixation time show that the main effect of color series type of nail images is significant $(F(3,56)=7.872$, $\mathrm{p}=0.000$ ). Univariate ANOVA is performed for fixation count, and the results show that the main effect of color series type on nail images is significant $(F(3,48)$ $=18.821, \mathrm{p}=0.000)$.

After paired comparison, it is found that the fixation time and count of the active consumers on the nail pictures of warm color series are significantly higher than those of other color series, and there is no significant difference in the fixation time among the neutral color series, cool color series and nude color series.

Specifically, based on the fixation time, the difference significance between warm color series and neutral color series is 0.001 , and it is the same as the significance between warm and cool color series, and that between warm and nude color series is 0.000 . The significance of neutral and cool color series is 0.948 , and that between neutral and nude color series is 0.557 . The difference between cool and nude color series is 0.602. (As shown in table 1).
Based on the fixation count, the significance between the series of warm color and other color is 0.000 separately. The significance of neutral color series and cool color series is 0.100 , and that between neutral and nude color series is 0.788 . The significance between cool and nude color series is 0.057. (As shown in table 2).

Table 1. Paired comparison of fixation time of active personality on four color series

\begin{tabular}{cccc}
\hline & neutral & cool & nude \\
\hline warm & 0.001 & 0.001 & 0.000 \\
neutral & & 0.948 & 0.557 \\
cool & & & 0.602
\end{tabular}

Table 2. Paired comparison of fixation count of active personality on four color series

\begin{tabular}{cccc}
\hline & neutral & cool & nude \\
\hline warm & 0.000 & 0.000 & 0.000 \\
neutral & & 0.100 & 0.788 \\
cool & & & 0.057
\end{tabular}

The paired comparison of fixation time and fixation count shows that the color series of nail images has a significant effect on the fixation time and fixation count, for the individuals with the characteristics of active personality.

\section{Fixation time}

After pointwise comparison of fixation time among the four color series, it is found that there is a significant difference in fixation time between nail images of warm color and neutral color $(\mathrm{t}=3.027, \mathrm{p}=0.006)$. There is a significant difference in the fixation time of nail images between warm colors and cool colors $(\mathrm{t}=3.061, \mathrm{p}=0.005)$. There is a significant difference in fixation time between warm and nude nail images $(\mathrm{t}=3.922, \mathrm{p}=0.001)$.

Table 3. Mean fixation time of active personality on four color series

\begin{tabular}{cccc}
\hline Color type & N & Mean & SD \\
\hline warm & 13 & 4029.131 & 2131 \\
neutral & 13 & 1788.177 & 1607 \\
cool & 13 & 1745.085 & 1641 \\
nude & 13 & 1402.069 & 1135
\end{tabular}

The comparison results of fixation time show that the active consumers pay more attention to the warm color nail pictures and the fixation time is longer than others. The fixation preference of neutral, cool and nude colors are not significant. 


\section{Fixation count}

After pointwise comparison of fixation count of the four color series, it is found that there is a significant difference in fixation count between nail images of warm color series and neutral color series $(t=6.703, p=0.000)$. There is a significant difference in the fixation count of nail images between warm color series and cool color series $(\mathrm{t}=3.746, \mathrm{p}=0.001)$. There is a significant difference in fixation count between warm and nude color series nail images $(\mathrm{t}=7.314, \mathrm{p}=0.000)$.

Table 4. Average fixation count of active personality on four color series

\begin{tabular}{cccc}
\hline Color type & $\mathrm{N}$ & Mean & SD \\
\hline warm & 13 & 25.932 & 8.05 \\
neutral & 13 & 7.846 & 5.44 \\
cool & 13 & 13 & 9.47 \\
nude & 13 & 7.007 & 4.62
\end{tabular}

The fixation preference of neutral, cool and nude colors is not significant.

In conclusion, hypothesis 1 is supported. Active personality prefers warm color series (red, orange, yellow). The fixation time and fixation count of the active participants on the warm color series are significantly higher than those of other series.

Taking consumers with strength personality type as the research object, univariate ANOVA is conducted on fixation time, and the results show that the main effect of color series type on nail images is not significant ( $F$ $(3,32)=1.223, p=0.317)$. The results of one-way ANOVA of fixation count show that the main effect of nail color type is not significant $(F(3,32)=1.181$, $\mathrm{p}=0.332$ ). To conclude, the color series of nail images has no significant effect on the fixation time and fixation count among individuals with strength personality characteristics. Hypothesis 2 is not supported.

Taking consumers with perfect personality type as the research object, univariate ANOVA is conducted on fixation time, and the results show that the main effect of color series type on nail images is not significant $(\mathrm{F}$ $(3,32)=1.271, p=0.301)$. The results of one-way ANOVA of fixation count show that the main effect of color series type on nail images is not significant ( $F$ $(3,32)=1.564, p=0.217)$. To conclude, the color series of nail images has no significant effect on the fixation time and fixation count among individuals with perfect personality characteristics. Hypothesis 3 is not supported.

Taking consumers with peaceful personality type as the research object, univariate ANOVA is conducted on fixation time, and the results show that the main effect of color series type of nail images is not significant $(\mathrm{F}(3,28)$ $=1.488, \mathrm{p}=0.239)$. The results of one-way ANOVA of fixation count show that the main effect of color series type on nail images is not significant $(\mathrm{F}(3,28)=1.279$, $\mathrm{p}=0.301$ ). To conclude, the color series of nail images has no significant effect on the fixation time and fixation count among individuals with peaceful personality traits. Hypothesis 4 is not supported.

\subsection{The Attractiveness of Four Color Series to Four Personality Types}

The entry time refers to the time when the participants first notice the target. The shorter the staring time is, the higher the participants' attention to the target object will be and the more attention that they pay to the target [15]. The shorter the time it takes consumers to focus on the interest area, the more attractive the interest area will be to consumers.

As shown in table 5. Regardless of the personality type of consumers, the entry time of warm color series is lower than that of other color series, followed by cool and neutral color series. In addition, the entry time of nude color series is the longest. In other words, it takes consumers the least time to find the interest area composed of warm color series. The warm color series are the most attractive to consumers as well.

Table 5. The entry time mean distribution (unit: $\mathrm{ms}$ )

\begin{tabular}{lrlll}
\hline & warm & cool & neutral & nude \\
\hline active & 651 & 860 & 929 & 1301 \\
strength & 510 & 680 & 728 & 1005 \\
perfect & 461 & 597 & 638 & 874 \\
peace & 452 & 600 & 649 & 917
\end{tabular}

After Two-way Repeated Measures ANOVA and analysis of entry time, it is found that the main effect of personality type is not significant. The main effect of color series type is significant $(\mathrm{F}(3,165)=168.432$, $\mathrm{p}=0.000)$. This indicates that there are significant differences in the visual attraction ability of the four color series to consumers. Further analysis shows that color series has a significant main effect on consumers, no matter they are active, strength, perfect or peaceful personality type.

The results show that the entry time of warm color series is the shortest, followed by the cool color series and neutral color series, and the longest is the nude color series. This indicates that the color has a sequential effect on the eyeball attractiveness of consumers, and the order is warm color series, cool color series, neutral color series and nude color series. Thus hypothesis 5 is supported.

The results show that consumers of the four personality types have various preferences for the four nail color series. The data of fixation time and fixation count of nail images with four color series show that the 
fixation preference of active consumers for nail images with warm color series, is significantly higher than that of the other. There is no significant difference in the fixation of color nail images among strength, peaceful and perfect consumers. The data of the entry time of consumers to four color series pictures shows that the attractive attributes of color belongs to the physiological existence and does not change with consumers' preference. The sequence of attractiveness is warm, cool, neutral and nude. Hypothesis 5 is supported.

\section{CONCLUSION}

The theoretical implications of this research are as follows. This research comprehensively discusses what kind of color would be more attractive for four personality types, which can provide more specific improvement measures and suggestions to enhance the service quality. To some extent, this research improves the theoretical approach to the service quality by analyzing consumer psychology, and provides theoretical decision support for service industry to improve the service quality.

The practical implications of this study are as follows. First, service personnel can infer the consumer personality type according to the consumers' behavior and recommend the commodities that conform to their color preference, which in turn is helpful to achieve the purpose of promoting transaction and stimulating consumption. Second, in practice, the advertising colors with warm and cool colors will attract consumers' attention and maximize the advertising effect in the marketing planning of the target market composed of female groups. Third, the individual who habitually uses warm color perform lively behavior characteristic, to some certain extent, which has certain enlightenment to the work of marketing promotion, such as personalized recommendations online or offline.

Although we try to avoid the influence of other factors on the experimental results by controlling various interference variables, there are certain limitations in this study. First, the study did not incorporate the rational or emotional thinking of consumers into the model for research. Second, all the participants are college females. It is necessary to increase the diversity of sample groups to make the research more universal. Third, the research finding that the preference effect of other personality type is not obvious except active personality.

\section{AUTHORS' CONTRIBUTIONS}

[1] Baoku Li, Bo Zhao, Ying Liu,Tingting Guo. Analysis of rural residents' online consumption willingness to pay[J].Management World, 2018, 34 (06): 94-103.

[2] Baoku Li, Bo Zhao, Tingting Guo. Impact of negative online reviews and sources on consumers' willingness to buy - The Regulatory Effects of Product Categories[J]. Technology and Economics, 2019, 32 (03): 96-99-105.

[3] Zhengxiang Wu, Baoku Li, Bo Zhao. Impact of Retailer Altruistic Preferences on Brand Competition and Channel Competition Coexistence Supply Chain Decisions[J].Soft Science, 2017, 31 (11): 110-116-122.

[4] Baoku Li, Zhengxiang Wu, Bo Zhao, Xiaoqiang Zhang. Information asymmetry under the conditions of outsourced after-sales service channel interest game research[J].Management World, 2016 (01): 182-183.

\section{ACKNOWLEDGMENTS}

I would like to express my gratitude to all those who have helped me during the writing of this thesis. I gratefully acknowledge the help of my supervisor Professor Li. I do appreciate his patience, encouragement, and professional instructions during my thesis writing Last, my gratitude also extends to my family who have been assisting, supporting and caring for me all of my life.

\section{REFERENCES}

[1] Su, Y., Fang, L.Z., and Chen, Y.Y. (2018), "The Origin and Development of Brand EmotionBased on Emotional Marketing Literature Review"[J]. China Business \& Market, Vol. 32 No.6, pp. 53-61. (in Chinese)

[2] Omer, Artun., Levin, D. and Wiley. (2015), Predictive Marketing: Easy Ways Every Marketer Can Use Customer Analytics and Big Data.

[3] Smith, G.P.C. (1959), "A system of colorpreferences", The American Journal of Psychology, Vol. 72 No.4, pp. 487-502.

[4] Marzolf, S.S. and Kirchner, J.H. (1973), "Personality traits and color choices for house tree person drawings", Journal of Clinical Psychology, Vol. 29 No.2, pp. 240-245.

[5] Granger, G.W. (1955). "The prediction of preference for color combinations. Journal of General Psychology," Vol. 52 No.2, pp. 213-222.

[6] Fang, F., Su, Y.Y., and Li, W. (2015), "Research on the underwear's color psychological cognition habit of youth groups based on the eye tracking technology", Journal of Donghua University, Vol. 71 No.1, pp. 169-177.

[7] Duan, W.J., Xie, D., Li, L., and Hu, W.P. (2016), "Status quo, difficulties and solutions of research on personality advantage and virtue", Psychological science, No.4, pp. 985-991. (in Chinese) 
[8] Wang, H.Z., Jiang, Q. and Hou, L.L. (2017), 'Influence of college students' personality advantages on subjective well-being: the use of advantages and the role of stressful sexual events", Psychological development and education, Vol. 33 No.1, pp. 95-104. (in Chinese)

[9] Ma, H.Y. and Shen, M. (2012), "Empirical research on employee characteristics, marketing ability and marketing performance", Modern management science, No.7, pp. 108-110. (in Chinese)

[10] Li, W.L. (2014), "The discourse of color visual transmission in packaging design", Packaging Engineering, No.12, pp. 104-107.

[11] Chen, L.B., Zhang, X.L., and Zhang, J.Y. (2016), "Influence of consumer color preference on brand personality construction", Enterprise economics, No. 3, pp. 18-21. (in Chinese)

[12] Hamel, S., Guyader, N., Pellerin, D., and Houzet, D. (2016), "Contribution of color in saliency model for videos", Signal Image \& Video Processing, Vol. 10 No.3, pp. 423-429.

[13] Livingston, L.A., Sandals, L.H. and Vickers, J.N. (1992), "Monitoring the effect of color on performance in an instructional gaming environment through an analysis of eye movement behaviors", Journal of Research on Computing in Education, Vol. 25 No.2, pp. 233-242.

[14] Wu, D.J. and Lohse, G.L. (2001), "Eye movement patterns on chinese yellow pages advertising", Electronic Markets, Vol. 11 No.2, pp. 87-96.

[15] Pang, Y.Y. (2015), "Study on the Eye Movement Evaluation of Color Preference of Small Household Appliances", Packaging Engineering, Vol. 36 No.4, pp. 97-100. (in Chinese) 